\title{
Structural insight into the mechanism of neuraminidase inhibitor-resistant mutations in
}

\section{human-infecting H10N8 Influenza A virus}

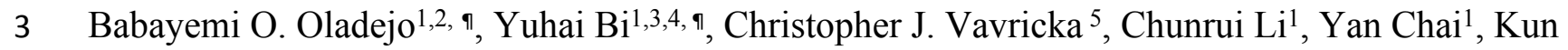

$4 \mathrm{Xu}^{2,6}$, Liqiang $\mathrm{Li}^{7,8}$, Zhe $\mathrm{Lu}^{7,9}$, Jiandong $\mathrm{Li}^{7,8}$, Gary Wong ${ }^{1,3}$, Sankar Mohan ${ }^{10}$, B. Mario Pinto ${ }^{10}$,

5 Haihai Jiang ${ }^{1,11}$, Jianxun $\mathrm{Qi}^{1}$, George Fu Gao ${ }^{1,2,3,4,11,12}$, Po Tien ${ }^{1,2, *}$ and Yan $\mathrm{Wu}^{3,6, *}$

7 1. CAS Key Laboratory of Pathogenic Microbiology and Immunology, Institute of Microbiology,

8 Chinese Academy of Sciences, Beijing 100101, China

9 2. University of Chinese Academy of Sciences, Beijing 100049, China

10 3. Shenzhen Key Laboratory of Pathogen and Immunity, Shenzhen Third People's Hospital,

11 Shenzhen 518112, China

12 4. Center for Influenza Research and Early-warning (CASCIRE), Chinese Academy of Sciences,

13 Beijing 100101, China

14 5. Graduate School of Science, Technology and Innovation, Kobe University, Kobe Japan

15 6. Research Network of Immunity and Health (RNIH), Beijing Institutes of Life Science, Chinese

16 Academy of Sciences, Beijing 100101, China

17 7. BGI-Shenzhen, Shenzhen 518083, China

18 8. China National Genebank, BGI-Shenzhen, Shenzhen 518083, China

19 9. Qingdao University-BGI Joint Innovation College, Qingdao University, Qingdao 266071, China 
20 10. Department of Chemistry, Simon Fraser University, Burnaby, BC, Canada

21 11. College of Veterinary Medicine, China Agricultural University, Beijing 100193, China

22 12. National Institute for Viral Disease Control and Prevention, Chinese Center for Disease

23 Control and Prevention (China CDC), Beijing 102206, China

24

$25 *$ Corresponding authors

26 E-mail: wuy@biols.ac.cn, tienpo@,im.ac.cn

27

28 १These authors contributed equally to this work. 


\section{Abstract}

30 The emergence of drug resistance in avian influenza virus (AIV) is a serious concern for public

31 health. Neuraminidase (NA) isolated from a fatal case of avian-origin H10N8 influenza virus

32 infection was found to carry a drug-resistant mutation, NA-Arg292Lys (291 in N8 numbering). In

33 order to understand the full potential of $\mathrm{H} 10 \mathrm{~N} 8$ drug resistance, the virus was first passaged in the

34 presence of the most commonly used neuraminidase inhibitors (NAIs), oseltamivir and zanamivir.

35 As expected, the Arg292Lys substitution was detected after oseltamivir treatment, however a novel

36 Val116Asp substitution (114 in N8 numbering) was selected by zanamivir treatment. Next

37 generation sequencing (NGS) confirmed that the mutations arose early (after passages 1-3) and

38 became dominant in the presence of the NAI inhibitors. Extensive crystallographic studies

39 revealed that N8-Arg292Lys resistance results mainly from loss of interactions with the inhibitor

40 carboxylate, while rotation of Glu276 was not impaired as observed in the N9-Arg292Lys, a group

412 NA structure. In the case of Val116Asp, the binding mode between oseltamivir and zanamivir is

42 different. Asp151 forms stabilized hydrogen bond to guanidine group of zanamivir, which may

43 compensate the resistance caused by Val116Asp. By contrast, the amino group of oseltamivir is

44 too short to maintain this hydrogen bond, which result in resistant. Moreover, the oseltamivir-

45 zanamivir hybrid inhibitor MS-257 displays higher effectiveness to Val116Asp than oseltamivir,

46 which support this notion. 


\section{Author Summary}

48 Aside from vaccination, NAIs are currently the only alternative for the clinical treatment and

49 prophylaxis of influenza. Understanding the mechanisms of resistance is critical to guide in drug

50 development. In this study, two drug-resistant NA substitutions, Val116Asp and Arg292Lys, were

51 discovered from oseltamivir and zanamivir treatment of H10N8 virus. Crystal structural analyses

52 revealed two distinct mechanisms of these two resistant mutations and provide the explanation for

53 the difference in susceptibility of different NAIs. Zanamivir and laninamivir were more effective

54 against the resistant variants than oseltamivir, and Arg292Lys results in more serious oseltamivir

55 resistance in N9 than N8 subtype. This study is well-correlated to influenza pandemic/epidemic

56 pre-warning, as the discovery of inhibitor resistant viruses will help for new drug preparedness. 


\section{Introduction}

58 Four NAIs, oseltamivir, zanamivir, peramivir and laninamivir, are currently available for the

59 clinical treatment of influenza virus infections [1-4]. Oseltamivir has been extensively used due to

60 its high oral bioavailability [5], however resistance to both oseltamivir and peramivir is prevalent.

61 On the other hand, although zanamivir and laninamivir offer advantages in terms of drug

62 resistance, both inhibitors are highly polar in their active forms and therefore have lower

63 bioavailability. Furthermore, there are still many known substitutions that lead to zanamivir and

64 laninamivir resistance including Glu119Gly/Ala/Asp, Gln136Lys, Asp151Ala/Asn/Gly/Val,

65 Arg152Lys, Ile222Arg, Asp198Asn, Arg292Lys and Arg371Lys (N2 numbering) [6-10].

66 Resistance to NAIs usually results from substitutions of highly conserved amino acid residues that

67 form the NA active site. The influenza NA active site contains 8 conserved catalytic residues, and

68 an additional frame of 11 residues that provides structural support to the active site [11, 12].

69 Substitution of non/semi-conserved residues has also been shown to lead to NAI resistance [13].

70 Semi-conserved influenza NA residues such as Ile117 and Lys 150 have been observed to confer

71 NAI resistance in N1 subtype NA[14, 15], while Gln136 has been observed in both N1 [16] and

72 N2 [17] NA subtypes. However, the mechanisms of drug resistance related to semi-conserved

73 residues are poorly understood $[6,18,19]$.

74 In recent years, some new AIVs, either highly pathogenic (HPAIV) or low pathogen AIV

75 (LPAIV), have emerged with ability to infect humans in addition to H5N1 HPAIV[20-24], due to

76 extensive poultry transportation and wild bird migration [25]. LPAIV H10N8 human-infecting

77 cases were identified in Jiangxi Province, China in 2013 [24, 26]. In December 2013, A/Jiangxi-

78 Donghu/346/2013/H10N8 with the NA-Arg292Lys (N2 numbering, 291 in N8 numbering) 
mutation was discovered in the trachea aspirate of a 73-year-old patient who died 3 days following oseltamivir treatment [27]. Therefore, H10N8 and other avian-origin influenza A viruses possess a high potential for human pathogenicity and hence pose public health concern.

There are two phylogenetic groups of influenza A NAs (N1, N4, N5 and N8 belong to group 1, while N2, N3, N6, N7 and N9 belong to group 2) [28]. Arg292Lys has been widely reported in group 2 NAs (N2 and N9), while the report of Arg292Lys in a group 1 NAs has never been observed in nature. Structural analysis of N9-Arg292Lys has been shown to result in unfavorable Glu276 conformation for oseltamivir binding [10]. However, the mechanism of Arg292Lys resistance in a group $1 \mathrm{~N} 8$ is not well understood due to lack of crystal structures (apo and holo).

In this study, we explored the potential drug-resistant substitutions of A/JiangxiDonghu/346/2013/H10N8 by passaging the virus in the presence of zanamivir and oseltamivir, respectively. Besides the clinical Arg292Lys substitution which was identified after in vitro oseltamivir treatment, a novel Val116Asp substitution was also identified after zanamivir treatment. The residue 116 (residue 114 in N8 numbering) does not play a direct role in active site framework or drug binding and therefore the mechanism underlying Val116Asp resistance is perplexing. We have determined the binding capability $\left(\mathrm{K}_{\mathrm{m}}\right)$ of $\mathrm{N} 8$ wildtype and two substitutions. The data indicated that Val116Asp and Arg292Lys mutations reduce the affinity between the enzyme and substrate, which results from the conformational shift of the key residues in the active site. Moreover, the inhibition assay demonstrated that the oseltamivir resistance $(1,000$-fold $)$ caused by Arg292Lys in N8 is much less severe than that of H7N9 (100,000-fold). We have also determined the apo crystal structures of N8 wild type and the two mutants and the holo structures in complex with oseltamivir, zanamivir, peramivir and laninamivir. These crystal structures reveal 
101 the underlying mechanism by which Arg292Lys and Val116Asp mutations develop resistance

102 towards NAIs.

103

104

105

106

107

108

109

110

111

112

113

114

115

116

117

118

119

120

121

\section{Results}

\section{In vitro NAI Selective Pressure}

H10N8 virus was subjected to 2-fold increases in the concentration of oseltamivir $(80-10240 \mu \mathrm{M})$ and zanamivir (20-2560 $\mu \mathrm{M})$ over 8 passages. Prior quantification by plaque assays showed that the virus was resistant to oseltamivir at $13.3 \mu \mathrm{M}$ and zanamivir at $1.33 \mu \mathrm{M}$. After each passage, the hemagglutinin (HA) titer was determined, recorded in triplicate and used to estimate the concentration of virus to be seeded in the next passage. A negative HA titer was observed in the last passage indicating the absence of virus (S1 Table).

\section{Substitutions under NAI Pressure}

Viruses from each passage under inhibitor treatment were subject to high-throughput sequencing for whole genome substitution and intra-cell substitution analysis. The result showed that as expected, the drug-resistant Arg292Lys substitution in NA was detected after oseltamivir treatment; however, a novel NA Val116Asp substitution arose after zanamivir treatment, suggesting a direct role in zanamivir-resistance (S1 Fig). The Arg292Lys mutation under oseltamivir treatment and the Val116Asp mutation under zanamivir treatment arose and became dominant quickly (1-3 passages). Unexpectedly, the pre-exist frequency of Arg292Lys was found to be at $28 \%$ in the non-drug treatment control. However, just after 1 passage under oseltamivir treatment, it increased to $92 \%$ and levelled off at $92 \%$ throughout the next 6 oseltamivir treatment passages (Fig 1A and 2). For zanamivir treatment, the pre-exist frequency of Val116Asp was found 
122 to be at $2 \%$; after 1 passage, it increased quickly to $34 \%$, and became absolute dominant (90\%)

123 (Fig 1B and 2).

124 In addition to these two NA substitutions, several substitutions occurred on the HA and other 125 certain internal genes. Two HA substitutions, Lys167Glu and Ile413Thr, were pre-existing at the 126 frequency of $49 \%$ and $48 \%$ respectively, and both became dominant under oseltamivir/ zanamivir 127 treatment and kept steady along cell passages with the increase of drug concentration. Importantly, 128 Val139Ile in Polymerase basic protein 2 (PB2) arose and became dominant in the first passage of 129 zanamivir treatment, with the frequency of $81 \%$. However, this was not observed in oseltamivir 130 treatment passages. One common substitution Cys95Phe in Polymerase acidic protein (PA) 131 occurred in both treatment passages. PA Cys95Phe also pre-existed in the drug-free treatment with 132 frequency of $12 \%$, which showed a slow increasing trend and became dominant at passage 7 in 133 oseltamivir treatment. Meanwhile, in zanamivir treatment passages, it became absolute dominant 134 within 3 passages and levelled off. Another PA substitution Val91Leu was only observed in 135 oseltamivir treatment. This mutation pre-existed in the drug-free treatment control at a frequency 136 of $10 \%$ and showed a fluctuating trend along passages. Other two substitutions, HA Gly89Arg and 137 M1 Gln158Arg also showed fluctuating trends along oseltamivir treatment passages. Fluctuating 138 trends for HA Gly89Arg mutation was also observed in zanamivir treatment passages (S1 Fig and 139 Fig 2).

140 Collectively, we can find that most of these substitutions are pre-existing in the original NAI-free 141 cultures. These pre-existing substitutions might be the natural selection pools when under drug 142 treatment. Correlation analysis of these substitutions using their frequencies values found several 143 strong links: one negative link between HA 179Arg and M1 Gln158Arg, one negative link between 144 HA 179Arg and PA Cye95Phe along oseltamivir treatment; one positive link between NA 
145

146

147

148

149

150

151

152

153

154

155

156

157

158

159

160

161

162

163

164

165

166

V116Asp and 292Arg. These results suggest that synergy might exist along the dynamic changes of these substitutions, which will be further studied in the future.

\section{Reduced Substrate Affinity and Inhibition of N8-Arg292Lys and N8-Val116Asp}

NA was prepared according to the previously reported methods [29-31]. Lower substrate affinity was observed for N8-Arg292Lys (30-fold) and Val116Asp (6-fold) mutants when compared to the wildtype N8. The recombinant Val116Asp and Arg292Lys variants also exhibited reduced sensitivity to the four clinically used NAIs (oseltamivir, zanamivir, peramivir and laninamivir) relative to the wildtype. The Arg292Lys mutant exhibited higher resistance to all of the four NAIs compared to the Val116Asp mutant. Both mutants showed high resistance to oseltamivir, with a 926-fold and 128-fold increase in mean $\mathrm{IC}_{50}$ for Arg292Lys and Val116Asp, respectively. Val116Asp was moderately resistant to zanamivir (40-fold) while Arg292Lys displays much higher zanamivir resistance (704-fold). The resistance pattern of Val116Asp and Arg292Lys to laninamivir is similar to that of zanamivir, with 10 -fold and 90 -fold lower potencies, respectively.

On the other hand, Arg292Lys showed much higher resistance to peramivir with a 3,169-fold increase in mean $\mathrm{IC}_{50}$ value, while Val116Asp exhibited a moderate 20 -fold reduced sensitivity (Table 1). Interestingly, the oseltamivir-zanamivir hybrid inhibitor, MS-257 was found to be the least affected inhibitor by these mutations when compared to all four clinically used NAIs. The increase in the mean $\mathrm{IC}_{50}$ values of MS-257 against Arg292Lys and Val116Asp mutants were 19fold and 7-fold, respectively.

\section{Val116Asp and Arg292Lys Result in Subtle N8 Active Site Changes}

The crystal structures of native N8, Val116Asp and Arg292Lys were determined at resolutions of $1.9 \AA, 1.9 \AA$ and $1.6 \AA$, respectively (S2 Table). The apo structures of N8 wildtype, Val116Asp 
167

168

169

170

171

172

173

174

175

176

177

178

179

180

181

182

183

184

185

186

187 188 inhibitor.

and Arg292Lys showed similar overall active site arrangements, with some differences observed regarding Gln136, Thr148, Glu276 and Tyr406 (Fig 3). Specifically, the conformations of 150loop between N8 wildtype and Val116Asp show slightly different, because of the conformational change of Thr148 and Gln136. The conformations of 430-loop between N8 wildtype and Arg292Lys display variable configurations. Moreover, the side chain of Gln136, Arg118, Tyr406 and Glu276 between these two native structures exhibit different conformations. All these observed conformational differences explain why the two N8 mutants have lower affinity to the substrate compared to N8 wildtype.

\section{A Distinct Mechanism of Arg292Lys Drug Resistance in Group 1 N8}

To understand the mechanisms of N8 drug resistance, inhibitor complex structures with wildtype or mutant N8 were compared. The crystal structures of wildtype N8 complexed with zanamivir, oseltamivir, laninamivir and peramivir were determined at resolutions of $1.8 \AA, 2.1 \AA, 1.8 \AA$ and 2.0 A, respectively (S3 Table). N8-Arg292Lys complexes were determined at resolutions of 1.9 $\AA, 1.8 \AA, 2.0 \AA$ and $1.8 \AA$ for zanamivir, oseltamivir, laninamivir and peramivir, respectively (S4 Table). Binding of oseltamivir, zanamivir, peramivir and laninamivir to wildtype H10N8 NA resembles that of typical group 1 NA binding (Fig 4A, C, E and G), with differences observed regarding Tyr347 in both oseltamivir and laninamivir complex structures. In the wildtype N8 complexes with zanamivir, oseltamivir and laninamivir, as well as the Arg292Lys complex with zanamivir, Tyr347 hydrogen bonds with Arg371 and the inhibitor carboxylate. In the wildtype peramivir complex, as well as the Arg292Lys complexes with laninamivir, oseltamivir and peramivir, Tyr347 points away from the active site where it can no longer interact with the 
Arg292 is part of the NA tri-arginine cluster that forms strong ionic interactions with the firstgeneration NAI carboxylates. In the structures of N8-Arg292Lys complexed with the four NAIs, Lys292 interacts with the carboxylate group of NAIs by a bridging water molecule (Fig 4A, C, E and G). The Glu276 adopts similar conformation in N8-Arg292Lys-zanamivir and laninamivir complexes (Fig 4C and G), but slightly differ in oseltamivir and peramivir-complexes (Fig 4A and E).

The binding of oseltamivir to N8 (group 1 NA) and N9 (group 2 NA) was carefully compared (Fig 4B, D, F and H). The flexibility of residue Glu276 is observed in the N9-Arg292Lys oseltamivir complex structure, but only a slightly shift in the N8-Arg292Lys oseltamivir complex structure. Specifically, in N8-Arg292Lys, Glu276 rotates towards Arg224 for optimal oseltamivir binding, which results in a mild shift of the oseltamivir hydrophobic group (1.11 $\AA$ ). In contrast, the side chain of Glu276 in N9-Arg292Lys is oriented toward the oseltamivir carboxylate hydrophobic pentyloxy group, which is pushed $2.98 \AA$ away from the active site. Furthermore, Arg292Lys substitution in N8 has no effect on the hydrogen bonds between Glu276 and Arg224, while, in N9, this substitution results in loss of one hydrogen bond between Glu276 and Arg224 (Fig 4B). These differences help to explain the observation that the effect of N8-Arg292Lysoseltamivir resistance is 1000-fold whereas the effect of N9-Arg292Lys resistance is 100,000-fold [10].

Notably, in the structure of N8-Arg292Lys-zanamivir, the orientation of Tyr347 is the same as N8 wildtype, which forms hydrogen bond with the side chain of Arg371 (Fig 4C). However, the residue 347 in N9 is Aspartic acid, which side chain is not enough to form hydrogen bond with Arg371 (Fig 4D). Therefore, zanamivir shows better inhibition to N8 than N9 with Arg292Lys substitution (Table 1). 


\section{The guanidine group of zanamivir compensates the Val116Asp resistant N8 substitution}

212 In order to understand the mechanism of Val116Asp resulting in more severe resistance to

213 oseltamivir than zanamivir, the zanamivir and oseltamivir complex structures of N8-Val116Asp

214 were determined at resolutions of $1.9 \AA$ and $2.1 \AA$, respectively (S5 Table). The 150-loop of

215 inhibitor bound-N8 (Val116Asp) complexes exhibited closed conformation, in which the Asp151

216 interacts with inhibitors. When compared the oseltamivir complex-N8 wild type with that of the

217 Val116Asp mutant, it was found that the side chain of Asp151 in N8 wildtype hydrogen bonds to

218 C4-amino group of oseltamivir with a distance of $2.70 \AA$, while the corresponding distance in N8-

219 Val116Asp was found to be $3.34 \AA$. In the case of zanamivir complexed structures, oxygen atom

220 on the main chain of Asp151 makes hydrogen bond interactions with the guanidine group of

221 zanamivir, with distance of $2.88 \AA$ and $3.03 \AA$ in $\mathrm{N} 8$ wildtype and N8-Val116Asp mutant,

222 respectively. The hydrogen bond interactions of zanamivir guanidine group withAsp151 is less

223 affected by the Val116Asp substitution than that of oseltamivir amino group. Moreover, Tyr347

224 in these two Val116Asp complex structures are shifted away from both inhibitors, while Tyr347

225 hydrogen bonds with the zanamivir and oseltamivir carboxylates groups in wildtype N8 complex

226 structures (Fig 5). Interestingly, the oseltamivir-zanamivir hybrid inhibitor MS-257 showed better

227 inhibition to N8-Val116Asp than oseltamivir (Table1).

\section{Discussion}

229 Although the crystal structures of all 9 influenza A NA subtypes have been solved to date [10, 28,

$23030-37$, the influenza A virus is constantly adapting and many new variations are being discovered,

231 especially those with drug resistance. For example, the crystal structure analysis of Arg292Lys

232 mutant of group 2 N9 was completed in 1998 [38], yet the corresponding group 1 N8 structure 
233 reported here contains unique features which are clearly observed during oseltamivir binding.

234 These structural differences are also reflected in the $\mathrm{K}_{\mathrm{m}}$ values which increased 30-fold for N8235 Arg292Lys and 88-fold for H7N9 N9-Arg292Lys [10], relative to the corresponding wildtype 236 NAs.

237 The Val116Lys mutation was the most intriguing N8 substitution. It is challenging to predict a 238 precise mechanism for NAI resistance, because the site of mutation is distal from the active site 239 frame work. Prior to solving the crystal structures, we anticipated that the Val116Asp substitution 240 might affect the interaction between Arg118 and the carboxylate group of the inhibitor, however 241 contrary to expectation, the N8-Val116Lys complex structures clearly suggested that this is not 242 the case and the loss of Tyr347-inhibitor interaction as the resistance mechanism. Tyr347 is found 243 only in group 1 NAs which should contain the 150-loop cavity [28]. This residue was also observed 244 to be a key factor in explaining the slightly higher NAI inhibition observed in N5 (typical group 1 245 NA) [36] relative to pandemic 09N1 (atypical group 1 NA) [39] and 57N2 (group 2 NA) [31]. 246 Thus, we previously speculated that Tyr347 might compensate for the open 150-loop in regards to 247 substrate binding [31].

248 Val116Lys also resulted in an altered conformation of the 150-loop residue Thr148 (Fig 3). It is 249 possible that the further changes observed in the loop residues 146-148 upon oseltamivir binding 250 might affect the ionic interaction of Asp151 with the oseltamivir amino group (Fig 5). Despite the 251 moderate effect on NAI inhibition, the Val116Asp substitution also resulted in a 6-fold $\mathrm{K}_{\mathrm{m}}$ increase 252 relative to the wildtype (Table 1). This indicates that Val116Asp also interferes with substrate 253 binding. 
254 Zanamivir and laninamivir are more similar to the human sialic acid, $N$-acetylneuraminic acid,

255 than oseltamivir and peramivir, and therefore should be less susceptible to drug-resistance. Of the

2564 clinical NAIs, zanamivir bound to both mutants in the most optimal conformation, whereas

257 oseltamivir binding was the least optimal. Resistance of both N8 variants to zanamivir and

258 laninamivir was also lower than that of oseltamivir and peramivir.

259 Some questions remain from the present analysis, including why peramivir was the most potent

260 inhibitor of wildtype N8 despite lacking any Tyr347 interactions. Moreover, binding of the prodrug

261 laninamivir octanoate (CS-8958) to N8 was distinct from group $109 \mathrm{~N} 1$ and similar to group 2 N2,

262 which indicates that NAI binding is not always group specific.

263 In summary, our current study revealed the 4-clinical available NAI resistant substitutions for N8

264 and the underlying mechanisms have also been structurally delineated, which will help for next-

265 generation drug development guide for drug usage in future prewarning of H10N8 AIV human

266 infections.

267 Materials and Methods

268 Cells, Virus and NAIs. A549 cells were obtained from China Infrastructure of Cell Line

269 Resources, Beijing and were grown in Dulbecco Modified Eagle's Medium (DMEM) (Gibco by

270 Life Technologies Incorporation ${ }^{\mathrm{TM}}$, Grand Island, New York, USA) containing 5\% fetal bovine

271 sera (FBS) (Irvine Scientific). The human influenza A/Jiangxi-Donghu/346/2013(H10N8) virus of

272 avian origin was propagated in the allantoic cavity of 10-day-old fertilized specific-pathogen-free

273 (SPF) hen eggs (Beijing Vital River Laboratory Animal Technology Co., Ltd.) for 96 hours at

$27437^{\circ} \mathrm{C}$. A hemagglutination assay was carried out on the harvested infected allantoic fluid in 96-

275 well plates. After adding $25 \mu \mathrm{L}$ of PBS to each well, $25 \mu \mathrm{L}$ of virus suspension was added to the 
276 first well, followed by a series of 2 -fold dilutions and gentle mixing. Next, about $25 \mu \mathrm{L}$ of freshly

277 prepared 1\% chicken RBC was added to each well and left for 30 minutes at room temperature.

278 Clear hemagglutination of the allantoic fluid containing the virus with chicken RBC indicates viral

279 growth and confirms the presence of the virus. Influenza neuraminidase inhibitors oseltamivir acid 280 (GS 4071) and zanamivir were synthesized at MedChem Express LLC, NJ, USA. MS-257 was 281 provided by cooperator Prof. Mario Pinto.

Isolation of H10N8 Variants with Decreased Susceptibility to Oseltamivir and Zanamivir. virus at a low multiplicity of infection (MOI 0.001 PFU per cell) in maintenance medium. The virus was allowed to adsorb for only 15 minutes at $37^{\circ} \mathrm{C}$, after which the inoculum was removed, and the cells were washed twice with pre-warmed PBS followed by addition of $1 \mathrm{ml}$ of each drug preparation to the seeded infected cells. The plates were incubated at $37^{\circ} \mathrm{C}$ for 72 hours. The virus titer in each culture supernatant was determined by hemagglutination of chicken RBC. The culture supernatant that contained the minimal dose drug that still resulted in cytopathy and detectable HA titer was used as inoculum to infect new cell monolayers at a low MOI. The virus from that sample was then allowed to grow in the presence of a series of 2-fold oseltamivir and zanamivir dilutions.

292 The virus concentrations used in the selection protocol varied, depending on the HA titer in the preceding passage and about $1 \mathrm{ml}$ aliquot of viral stock culture supernatant from the preceding

294 passage was used to infect the fresh cell monolayer cells. The HA titer was determined after each passage to estimate the amount of virus needed for infection. This selection was carried out for a total of 8 passages for both inhibitors in triplicates. Drug concentration ranged from $80 \mu \mathrm{M}$ to in triplicates. 
Virus RNA Extraction and Next-Generation Sequencing (NGS). RNA was extracted from virus isolates using a QIAamp viral RNA Mini Kit from QIAGEN, Germany (Cat. No. 52904). One step RT-PCR Kit Ver. 2 (TAKARA BIO INC. Cat. \#RR055A v201309Da, Japan) with slight modifications. Each RNA preparation was mixed with the enzyme mix in a $50 \mu \mathrm{L}$ reaction system. The thermal cycle parameters were $50^{\circ} \mathrm{C}$ for $30 \mathrm{mins}, 94^{\circ} \mathrm{C}$ for $2 \mathrm{mins}$, and then 35 cycles at $94^{\circ} \mathrm{C}$ for $30 \mathrm{secs}, 58^{\circ} \mathrm{C}$ for $30 \mathrm{secs}$, and $72^{\circ} \mathrm{C}$ for 3 mins 20 secs. Primers used were $20 \mu \mathrm{M}$ MBTuni-12 products by end-repairing, dA-tailing, adapter ligation and PCR amplification, according to the manufacturer instructions (Life technologies). The libraries were sequenced on an Ion Proton ${ }^{\mathrm{TM}}$

312 System, and sequencing depth was $1 \mathrm{G}$ base per sample. After sequencing, raw NGS short reads

313 were processed by filtering out low-quality reads, adaptor-contaminated reads (with $>15$ bp 314 matched to the adapter sequence), poly-Ns (with 8Ns) (SOAP2 (v2.21), <5 mismatches). Clean 315 short reads were then mapped onto the eight reference genome segments (A/Jiangxi-

317 (https://github.com/iontorrent/TS/tree/master/Analysis/TMAP) with a match rate larger than 0.90, 318 producing the short-read-reference-genome mapping file (SRRG file), and the dominant base on 319 each site was called to obtain the consensus genome. Consensus sequence alignments were done 320 using MUSCLE [40] to identify the variable sites. The consensus nucleotide sites, which have 321 changed in more than any one sample comparing with the original genome (A/Jiangxi- 
Donghu/346/2013 (H10N8)), are designated as variable sites. Intra-host single nucleotide variations (iSNV) and their correlations were analyzed based on the SRRG file.

Expression and Purification of Influenza Virus NA. Recombinant NA protein was prepared using the established baculovirus expression system $(19,20,22)$. The ectodomain (residues 81 to 469 in N8 numbering) of A/JD/346/2013/H10N8 was cloned into pFastBac1 baculovirus transfer vector (Invitrogen) and the Val114Asp and Arg291Lys substitutions were constructed by sitedirected mutagenesis PCR based on N8 wildtype and expressed in a baculovirus system for structural and functional analysis. A GP67 signal peptide was added at the $\mathrm{N}$ terminus to facilitate secretion of the recombinant protein, followed by a His tag, a tetramerizing sequence, and a thrombin cleavage site. Recombinant pFastBacl plasmid was used to transform $\mathrm{DH}_{10} \mathrm{Bac}^{\mathrm{TM}}$ Escherichia coli (Invitrogen). The recombinant baculovirus was obtained following the manufacturer's protocol, and Hi5 cell suspension cultures were infected with high-titer recombinant baculovirus. After growth of the infected Hi5 suspension cultures for 2 days, centrifuged media were applied to a 5-mL HisTrap FF column (GE Healthcare), which was washed with $20 \mathrm{mM}$ imidazole. The NA was thereafter eluted using $300 \mathrm{mM}$ imidazole. After dialysis and thrombin digestion ( $3 \mathrm{U} / \mathrm{mg}$ NA; BD Biosciences) overnight at $4{ }^{\circ} \mathrm{C}$, gel filtration chromatography was performed with a Superdex ${ }^{\circledR} 200$ 10/300 GL column (GE Healthcare) using 20 mM Tris-HCl and $50 \mathrm{mM} \mathrm{NaCl}(\mathrm{pH} \mathrm{8.0)}$ ) buffer or PBS buffer for crystallization or functional assay, respectively. Pure NA fractions were selected and further concentrated using a $10 \mathrm{kDa}$ (Millipore) membrane concentrator.

Crystallization, Drug-Soaking and Crystal Structure Determination. wildtype and mutant N8 crystals were obtained using the sitting-drop vapor diffusion method. The NA proteins $[1 \mu \mathrm{L}$ of $10 \mathrm{mg} / \mathrm{mL}$ protein in $20 \mathrm{mMTris}$ and $50 \mathrm{mMNaCl}(\mathrm{pH} 8.0)]$ were mixed with $1 \mu \mathrm{L}$ of reservoir 
solution. N8 wildtype crystals were obtained in the condition of $0.1 \mathrm{M}$ sodium acetate trihydrate pH 4.7 and 5\% w/v Polyethylene glycol 10,000. N8-Val116Asp crystals were obtained in the condition of 0.1M DL-malic acid pH 7.0 and 12\% Polyethylene glycol 3,350. N8-Arg292Lys crystals were obtained in the condition of $0.1 \mathrm{M}$ potassium phosphate monobasic/sodium phosphate dibasic pH 6.2 and 10\% Polyethylene glycol, 3,000.

All NA crystals were cryoprotected in mother liquor with the addition of $20 \%$ (vol/vol) glycerol before being flash-cooled at $100 \mathrm{~K}$ for obtaining apo structures. The crystals were then incubated in the mother liquor containing $20 \mathrm{mM}$ inhibitors (oseltamivir acid, zanamivir, peramivir, and laninamivir) and then flash-cooled at $100 \mathrm{~K}$.

Diffraction data were collected at Shanghai Synchrotron Radiation Facility beamline BL17U. The collected intensities were indexed, integrated, corrected for absorption, and scaled and merged using HKL-2000 (27). The structure of N8 was solved by molecular replacement using Phaser (28) from the CCP4 program suite (29), with the structure of N8 (PDB ID code 2HT7) as a search model. N8-Val116Asp and Arg292Lys were equally solved using the native N8 as the search model. The initial model was refined by rigid body refinement using REFMAC5 (30), and extensive model building was performed using COOT (31). Further rounds of refinement were performed using the phenix.refine program implemented in the PHENIX package (32) with energy minimization, isotropic ADP refinement and bulk solvent modeling. The stereochemical quality of the final model was assessed with PROCHECK (33). Structures were aligned and analyzed using PyMOL.

Fluorescent NA Activity Assay. A 4-methylumbelliferyl-Neu5ac-(MUNANA)-based fluorometric NA assay (34) was used for measuring the NA activity and inhibition. The 
367

368

369

370

371

372

373

374

375

376

377

378

379

380

381

382

383

384

385

386

387

388

389

390

391

392

393

394

395

appropriate protein concentrations were chosen after several rounds of preliminary tests. The $\mathrm{km}$ values for the active NAs were determined by mixing $10 \mu \mathrm{L}$ of each recombinant protein with 10 $\mu \mathrm{L}$ of buffer MES-CaCl2 buffer (pH 6.0) in each 96-well plate and serial dilutions $(9.76 \mu \mathrm{M}-$ $5 \mathrm{mM})$ of MUNANA (30 $\mu \mathrm{L})$ were added to each well. The fluorescence intensity of the released product was measured every 30 seconds for 1 hour at $37^{\circ} \mathrm{C}$ on the SpectraMax M5 (Molecular Devices), with excitation and emission wavelengths of $355 \mathrm{nM}$ and $460 \mathrm{nM}$, repectively. For inhibition assays, $10 \mu \mathrm{L}$ of recombinant protein was mixed with $10 \mu \mathrm{L}$ of PBS or inhibitor in 96well standard opaque plate and $30 \mu \mathrm{L}$ of $167 \mu \mathrm{M}$ MUNANA (Sigma, USA) in $33 \mathrm{mM}$ MES and 4 $\mathrm{mM} \mathrm{CaCl} 2(\mathrm{pH}$ 6.0) for a final substrate concentration of $100 \mu \mathrm{M}$. The inhibitors (in different concentrations) were pre-incubated with the NA protein for 30 minutes at $37^{\circ} \mathrm{C}$ before adding MUNANA, and then loaded on the SpectraMax ${ }^{\circledR}$ M5 Molecular devices. Fluorescence was monitored immediately after substrate addition at 1-minute intervals for 30 minutes. All assays were performed in triplicates and $\mathrm{IC}_{50}$ values for each inhibitor were calculated with Graphpad Prism 5.0 as the concentration of inhibitor resulting in a 50\% reduction in fluorescence units (FU) compared with the control.

\section{References}

1. He G, Massarella J, Ward P. Clinical pharmacokinetics of the prodrug oseltamivir and its active metabolite Ro 64-0802. Clin Pharmacokinet. 1999;37(6):471-84. Epub 2000/01/11. doi: 10.2165/00003088-199937060-00003. PubMed PMID: 10628898.

2. Greengard O, Poltoratskaia N, Leikina E, Zimmerberg J, Moscona A. The anti-influenza virus agent 4-GU-DANA (zanamivir) inhibits cell fusion mediated by human parainfluenza virus and influenza virus HA. J Virol. 2000;74(23):11108-14. Epub 2000/11/09. PubMed PMID: 11070006; PubMed Central PMCID: PMCPMC113191.

3. Birnkrant D, Cox E. The Emergency Use Authorization of peramivir for treatment of 2009 H1N1 influenza. N Engl J Med. 2009;361(23):2204-7. Epub 2009/11/04. doi: 10.1056/NEJMp0910479. PubMed PMID: 19884645.

4. Kubo S, Tomozawa T, Kakuta M, Tokumitsu A, Yamashita M. Laninamivir prodrug CS8958, a long-acting neuraminidase inhibitor, shows superior anti-influenza virus activity after a single administration. Antimicrob Agents Chemother. 2010;54(3):1256-64. Epub 
2010/01/06. doi: 10.1128/AAC.01311-09. PubMed PMID: 20047917; PubMed Central PMCID: PMCPMC2825999.

5. Davies BE. Pharmacokinetics of oseltamivir: an oral antiviral for the treatment and prophylaxis of influenza in diverse populations. J Antimicrob Chemother. 2010;65 Suppl 2:ii5-ii10. Epub 2010/03/20. doi: 10.1093/jac/dkq015. PubMed PMID: 20215135; PubMed Central PMCID: PMCPMC2835511.

6. Hurt AC, Holien JK, Parker M, Kelso A, Barr IG. Zanamivir-resistant influenza viruses with a novel neuraminidase mutation. J Virol. 2009;83(20):10366-73. Epub 2009/07/31. doi: 10.1128/JVI.01200-09. PubMed PMID: 19641000; PubMed Central PMCID: PMCPMC2753113.

7. Eshaghi A, Patel SN, Sarabia A, Higgins RR, Savchenko A, Stojios PJ, et al. Multidrugresistant pandemic (H1N1) 2009 infection in immunocompetent child. Emerg Infect Dis. 2011;17(8):1472-4. Epub 2011/08/02. doi: 10.3201/eid1708.102004. PubMed PMID: 21801626; PubMed Central PMCID: PMCPMC3381550.

8. Sheu TG, Deyde VM, Okomo-Adhiambo M, Garten RJ, Xu X, Bright RA, et al. Surveillance for neuraminidase inhibitor resistance among human influenza A and B viruses circulating worldwide from 2004 to 2008. Antimicrob Agents Chemother. 2008;52(9):3284-92. Epub 2008/07/16. doi: 10.1128/AAC.00555-08. PubMed PMID: 18625765; PubMed Central PMCID: PMCPMC2533500.

9. Yen HL, Hoffmann E, Taylor G, Scholtissek C, Monto AS, Webster RG, et al. Importance of neuraminidase active-site residues to the neuraminidase inhibitor resistance of influenza viruses. J Virol. 2006;80(17):8787-95. Epub 2006/08/17. doi: 10.1128/JVI.00477-06. PubMed PMID: 16912325; PubMed Central PMCID: PMCPMC1563878.

10. Wu Y, Bi Y, Vavricka CJ, Sun X, Zhang Y, Gao F, et al. Characterization of two distinct neuraminidases from avian-origin human-infecting H7N9 influenza viruses. Cell Res. 2013;23(12):1347-55. doi: 10.1038/cr.2013.144. PubMed PMID: 24165891; PubMed Central PMCID: PMC3847574.

11. Kobasa D, Wells K, Kawaoka Y. Amino acids responsible for the absolute sialidase activity of the influenza A virus neuraminidase: relationship to growth in the duck intestine. J Virol. 2001;75(23):11773-80. Epub 2001/11/02. doi: 10.1128/JVI.75.23.1177311780.2001. PubMed PMID: 11689658; PubMed Central PMCID: PMCPMC114763.

12. Aoki FY, Boivin G, Roberts N. Influenza virus susceptibility and resistance to oseltamivir. Antivir Ther. 2007;12(4 Pt B):603-16. Epub 2007/10/20. PubMed PMID: 17944268.

13. McKimm-Breschkin JL, Williams J, Barrett S, Jachno K, McDonald M, Mohr PG, et al. Reduced susceptibility to all neuraminidase inhibitors of influenza H1N1 viruses with haemagglutinin mutations and mutations in non-conserved residues of the neuraminidase. J Antimicrob Chemother. 2013;68(10):2210-21. Epub 2013/06/14. doi: 10.1093/jac/dkt205. PubMed PMID: 23759505; PubMed Central PMCID: PMCPMC3772742.

14. Hurt AC, Leang SK, Speers DJ, Barr IG, Maurer-Stroh S. Mutations I1 17V and I117M and oseltamivir sensitivity of pandemic (H1N1) 2009 viruses. Emerg Infect Dis. 2012;18(1):109-12. doi: 10.3201/eid1801.111079. PubMed PMID: 22260817; PubMed Central PMCID: PMC3310118.

15. Ilyushina NA, Seiler JP, Rehg JE, Webster RG, Govorkova EA. Effect of neuraminidase inhibitor-resistant mutations on pathogenicity of clade 2.2 A/Turkey/15/06 (H5N1) influenza virus in ferrets. PLoS Pathog. 2010;6(5):e1000933. Epub 2010/06/05. doi: 
10.1371/journal.ppat.1000933. PubMed PMID: 20523902; PubMed Central PMCID: PMC2877746.

16. Kaminski MM, Ohnemus A, Staeheli P, Rubbenstroth D. Pandemic 2009 H1N1 influenza A virus carrying a Q136K mutation in the neuraminidase gene is resistant to zanamivir but exhibits reduced fitness in the guinea pig transmission model. J Virol. 2013;87(3):1912-5. Epub 2012/11/30. doi: 10.1128/JVI.02507-12. PubMed PMID: 23192869; PubMed Central PMCID: PMCPMC3554154.

17. Dapat C, Suzuki Y, Saito R, Kyaw Y, Myint YY, Lin N, et al. Rare influenza A (H3N2) variants with reduced sensitivity to antiviral drugs. Emerg Infect Dis. 2010;16(3):493-6. Epub 2010/03/06. doi: 10.3201/eid1603.091321. PubMed PMID: 20202427; PubMed Central PMCID: PMCPMC3322031.

18. Hurt AC, Selleck P, Komadina N, Shaw R, Brown L, Barr IG. Susceptibility of highly pathogenic $\mathrm{A}(\mathrm{H} 5 \mathrm{~N} 1)$ avian influenza viruses to the neuraminidase inhibitors and adamantanes. Antiviral Res. 2007;73(3):228-31. Epub 2006/11/23. doi: 10.1016/j.antiviral.2006.10.004. PubMed PMID: 17112602.

19. McKimm-Breschkin J, Trivedi T, Hampson A, Hay A, Klimov A, Tashiro M, et al. Neuraminidase sequence analysis and susceptibilities of influenza virus clinical isolates to zanamivir and oseltamivir. Antimicrob Agents Chemother. 2003;47(7):2264-72. Epub 2003/06/25. PubMed PMID: 12821478; PubMed Central PMCID: PMCPMC161875.

20. Wei SH, Yang JR, Wu HS, Chang MC, Lin JS, Lin CY, et al. Human infection with avian influenza A H6N1 virus: an epidemiological analysis. The Lancet Respiratory medicine. 2013;1(10):771-8. Epub 2014/01/28. doi: 10.1016/S2213-2600(13)70221-2. PubMed PMID: 24461756.

21. Wang X, Jiang H, Wu P, Uyeki TM, Feng L, Lai S, et al. Epidemiology of avian influenza A H7N9 virus in human beings across five epidemics in mainland China, 2013-17: an epidemiological study of laboratory-confirmed case series. Lancet Infect Dis. 2017;17(8):822-32. Epub 2017/06/07. doi: 10.1016/S1473-3099(17)30323-7. PubMed PMID: 28583578.

22. Gao R, Cao B, Hu Y, Feng Z, Wang D, Hu W, et al. Human infection with a novel avianorigin influenza A (H7N9) virus. N Engl J Med. 2013;368(20):1888-97. Epub 2013/04/13. doi: 10.1056/NEJMoa1304459. PubMed PMID: 23577628.

23. Yang ZF, Mok CK, Peiris JS, Zhong NS. Human Infection with a Novel Avian Influenza A(H5N6) Virus. N Engl J Med. 2015;373(5):487-9. Epub 2015/07/30. doi: 10.1056/NEJMc1502983. PubMed PMID: 26222578.

24. Zhang T, Bi Y, Tian H, Li X, Liu D, Wu Y, et al. Human infection with influenza virus A(H10N8) from live poultry markets, China, 2014. Emerg Infect Dis. 2014;20(12):20769. Epub 2014/11/27. doi: 10.3201/eid2012.140911. PubMed PMID: 25425075; PubMed Central PMCID: PMCPMC4257803.

25. Gao GF. Influenza and the live poultry trade. Science. 2014;344(6181):235. Epub 2014/04/20. doi: 10.1126/science.1254664. PubMed PMID: 24744345.

26. Wan J, Zhang J, Tao W, Jiang G, Zhou W, Pan J, et al. [A report of first fatal case of H10N8 avian influenza virus pneumonia in the world]. Zhonghua Wei Zhong Bing Ji Jiu Yi Xue. 2014;26(2):120-2. Epub 2014/02/15. doi: 10.3760/cma.j.issn.20954352.2014.02.013. PubMed PMID: 24524404.

27. Chen H, Yuan H, Gao R, Zhang J, Wang D, Xiong Y, et al. Clinical and epidemiological characteristics of a fatal case of avian influenza A H10N8 virus infection: a descriptive 
study. Lancet. 2014;383(9918):714-21. Epub 2014/02/11. doi: 10.1016/S01406736(14)60111-2. PubMed PMID: 24507376.

28. Russell RJ, Haire LF, Stevens DJ, Collins PJ, Lin YP, Blackburn GM, et al. The structure of H5N1 avian influenza neuraminidase suggests new opportunities for drug design. Nature. 2006;443(7107):45-9. Epub 2006/08/18. doi: 10.1038/nature05114. PubMed PMID: 16915235.

29. $\mathrm{Xu} \mathrm{X,} \mathrm{Zhu} \mathrm{X,} \mathrm{Dwek} \mathrm{RA,} \mathrm{Stevens} \mathrm{J,} \mathrm{Wilson} \mathrm{IA.} \mathrm{Structural} \mathrm{characterization} \mathrm{of} \mathrm{the} 1918$ influenza virus H1N1 neuraminidase. J Virol. 2008;82(21):10493-501. Epub 2008/08/22. doi: 10.1128/JVI.00959-08. PubMed PMID: 18715929; PubMed Central PMCID: PMCPMC2573172.

30. Li Q, Qi J, Zhang W, Vavricka CJ, Shi Y, Wei J, et al. The 2009 pandemic H1N1 neuraminidase N1 lacks the 150-cavity in its active site. Nat Struct Mol Biol. 2010;17(10):1266-8. Epub 2010/09/21. doi: nsmb.1909 [pii]. PubMed PMID: 20852645.

31. Vavricka CJ, Li Q, Wu Y, Qi J, Wang M, Liu Y, et al. Structural and functional analysis of laninamivir and its octanoate prodrug reveals group specific mechanisms for influenza NA inhibition. PLoS Pathog. 2011;7(10):e1002249. Epub 2011/10/27. doi: 10.1371/journal.ppat.1002249 PPATHOGENS-D-11-00921 [pii]. PubMed PMID: 22028647; PubMed Central PMCID: PMC3197600.

32. Sun X, Li Q, Wu Y, Wang M, Liu Y, Qi J, et al. Structure of influenza virus N7: the last piece of the neuraminidase "jigsaw" puzzle. J Virol. 2014;88(16):9197-207. doi: 10.1128/JVI.00805-14. PubMed PMID: 24899180; PubMed Central PMCID: PMC4136277.

33. Wu Y, Qin G, Gao F, Liu Y, Vavricka CJ, Qi J, et al. Induced opening of influenza virus neuraminidase N2 150-loop suggests an important role in inhibitor binding. Sci Rep. 2013;3:1551. doi: 10.1038/srep01551. PubMed PMID: 23531861; PubMed Central PMCID: PMC3609017.

34. Li Q, Qi J, Wu Y, Kiyota H, Tanaka K, Suhara Y, et al. Functional and structural analysis of influenza virus neuraminidase $\mathrm{n} 3$ offers further insight into the mechanisms of oseltamivir resistance. J Virol. 2013;87(18):10016-24. doi: 10.1128/JVI.01129-13. PubMed PMID: 23824808; PubMed Central PMCID: PMC3753999.

35. Vavricka CJ, Liu Y, Li Q, Shi Y, Wu Y, Sun YP, et al. Special features of the 2009 pandemic swine-origin influenza A H1N1 hemagglutinin and neuraminidase. Chinese Sci Bull. 2011;56(17):1747-52. PubMed PMID: ISI:000291259300001.

36. Wang M, Qi J, Liu Y, Vavricka CJ, Wu Y, Li Q, et al. Influenza A virus N5 neuraminidase has an extended 150-cavity. J Virol. 2011;85(16):8431-5. Epub 2011/06/10. doi: JVI.00638-11 [pii]. PubMed PMID: 21653672; PubMed Central PMCID: PMC3147963.

37. Wu Y, Wu Y, Tefsen B, Shi Y, Gao GF. Bat-derived influenza-like viruses H17N10 and H18N11. Trends in microbiology. 2014;22(4):183-91. Epub 2014/03/04. doi: 10.1016/j.tim.2014.01.010. PubMed PMID: 24582528.

38. Varghese JN, Smith PW, Sollis SL, Blick TJ, Sahasrabudhe A, McKimm-Breschkin JL, et al. Drug design against a shifting target: a structural basis for resistance to inhibitors in a variant of influenza virus neuraminidase. Structure. 1998;6(6):735-46. Epub 1998/07/10. PubMed PMID: 9655825. 
39. Wu Y, Vavricka CJ, Wu Y, Li Q, Rudrawar S, Thomson RJ, et al. Atypical group 1 neuraminidase $\mathrm{pH} 1 \mathrm{~N} 1-\mathrm{N} 1$ bound to a group 1 inhibitor. Protein Cell. 2015;6(10):771-3. Epub 2015/09/04. doi: 10.1007/s13238-015-0197-6. PubMed PMID: 26334400; PubMed Central PMCID: PMCPMC4598326.

40. Edgar RC. MUSCLE: a multiple sequence alignment method with reduced time and space complexity. BMC Bioinformatics. 2004;5:113. Epub 2004/08/21. doi: 10.1186/14712105-5-113. PubMed PMID: 15318951; PubMed Central PMCID: PMCPMC517706.

\section{Supporting Information Legends}

Table 1. $\mathrm{K}_{\mathrm{m}}$ and $\mathrm{IC}_{50}$ values for $\mathrm{N} 8$ wildtype and mutant proteins. The $\mathrm{IC}_{50}$ values and $95 \%$ confidence intervals (CIs) are provided.

S1 Fig. Consensus sequence variations of H10N8 virus under drug treatment. The first row represents the structural proteins of $\mathrm{H} 10 \mathrm{~N} 8$ and vertical numbers represent different amino acid sites. The consensus amino sites list default amino acids, dots represent no substitutions and question marks represents unknown amino acids due to sites that were not covered by short reads.

Osel: oseltamivir, Zan: zanamivir, and the numeral to the right of Osel and Zan are the passage numbers.

S1 Table. Inhibitor concentration and HA titer of passaged viruses. The inhibitor concentration and HA titer for eight passages are listed in the table. “-” means no hemagglutination.

S2 Table. Crystallographic data collection and refinement statistics of native N8 and N8 mutations.

S3 Table. Crystallographic data collection and refinement statistics of N8-inhibitor complexes.

S4 Table. Crystallographic data collection and refinement statistics of N8-Arg292Lys-inhibitor complexes. 
S5 Table. Crystallographic data collection and refinement statistics of N8-Val116Asp-inhibitor complexes

\section{Figure Legends}

Fig 1. Substitution frequency of Val116Asp and Arg292Lys in N8 under NAI pressure. (A) Mutation frequency of Arg292Lys increased sharply and throughout the passage period during oseltamivir treatment. (B) A similar trend was observed for Val116Asp during zanamivir treatment, although there was a reduction in the mutation rate at passages 5 and 7.

Fig 2. Heatmap and correlation network of oseltamivir treated samples. (A) Heatmap of amino acid residues under oseltamivir treatment from the 1st to 7 th passage. (B) Heatmap of amino acid residues under zanamivir treatment from the $1^{\text {st }}$ to $7^{\text {th }}$ passage. The values in each cell represent the substitution frequencies. A sudden change in the default frequency will lead to a change in the amino acid site. The ratio of the depth sequencing site or threshold of total depth is 10. Mutation frequency is based on color change. Each column represents the amino acid sites separated by "-" while each row represents the drug passage including positive control. POS: positive control, N/A: not available data to give a decisive result, OS: oseltamivir, Zan: zanamivir, and the numerals next to each inhibitor represent the passage numbers. (C) Correlation networks of different amino acid sites. (D) Correlation networks of different amino acid sites show a positive correlation between NA Asp116 and NA Arg292. To avoid error, smooth changing sites were used for correlation analysis which could be judged easily from the heatmap. Amino acid sites are indicated as different colors depending on the encoded protein. 2 or more amino acids connected together indicates a correlation larger than $0.8(\mathrm{r}>0.8)$. Correlations were determined using Pearson's rank correlation method. P-values of all correlations are less than 0.05 . Positive 
578 correlation is indicated by a red line connecting amino acids while negative correlation is indicated

579 by a green line.

Fig 3. Active site comparison of N8 wildtype (green), Val116Asp (cyan) and Arg292Lys

581 (magenta). (A) The comparison of the key residues conformation between N8 wildtype and N8-

582 Val116Asp. (B) Comparison of the key residues in the active site between N8 wildtype and N8-

583 Arg292Lys. Key residues are labeled in sticks.

Fig 4. Comparison of wildtype N8, N8-Arg292Lys and N9Arg292Lys binding to four NAIs.

585 (A and B) oseltamivir; $(\mathrm{C}$ and $\mathrm{D})$ zanamivir; $(\mathrm{E}$ and $\mathrm{F})$ peramivir and $(\mathrm{G}$ and $\mathrm{H})$ laninamivir. All

586 the inhibitors and the key residues are displayed in sticks. The hydrogen bonds are indicated in

587 dash. The PDB code of N9-Arg292Lys-oseltamivir, N9-Arg292Lys-zanamivir, N9-Arg292Lys-

588 peramivir and N9-Arg292Lys-laninamivir are 4MWW, 4MWX, 4MX0 and 4MWY, respectively.

Fig 5. Comparison of the binding mode between N8 wildtype and N8-Val116Asp with two

inhibitors. (A) The binding mode of N8 wildtype-oseltamivir (cyan) and N8-Val116Asp-

591 zanamivir (salmon). (B) The binding mode of N8 wildtype-zanamivir (bright yellow) and N8-

592 Val116Asp-zanamivir (orange). 
A $\quad$-Val116Asp

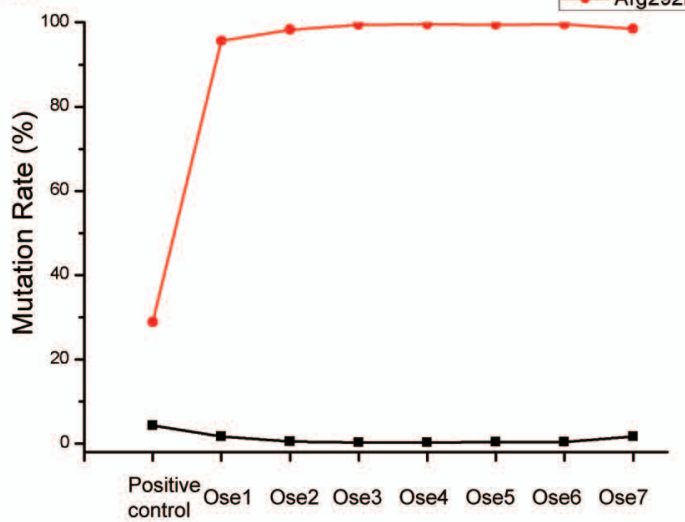

Virus Passages
B

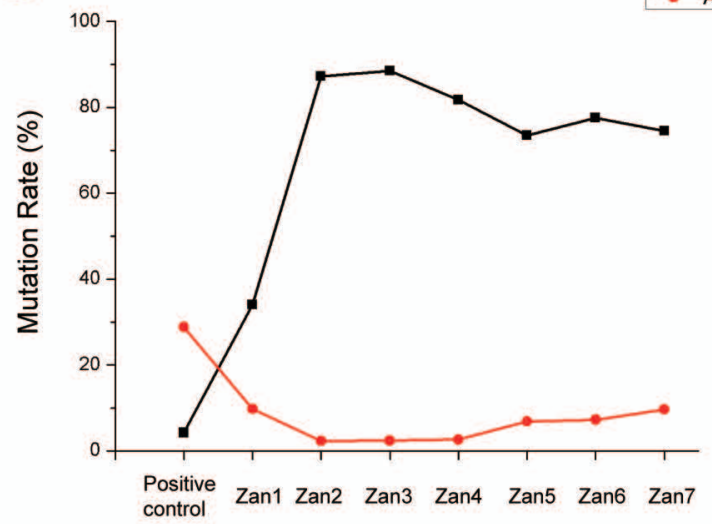

Virus Passages 


\begin{tabular}{|c|c|c|c|c|c|c|c|c|c|}
\hline 0.86 & 0.49 & & 0.48 & 0.10 & & 0.28 & 0.10 & 0.12 & POS \\
\hline 0.76 & & & & 0.22 & & & 0.08 & 0.10 & OS1 \\
\hline 0.49 & & 0.91 & & 0.14 & & & 0.09 & 0.45 & OS2 \\
\hline 0.61 & & 00 & & 0.03 & & & NA & NA & OS3 \\
\hline 0.84 & & 0.91 & & 0.10 & 0.84 & & 0.75 & 0.19 & OS4 \\
\hline 0.78 & & 0.74 & & 0.29 & & & 0.46 & 0.46 & OS5 \\
\hline 0.69 & & 0.73 & & 0.38 & NA & & NA & NA & OS6 \\
\hline 0.51 & & 0.66 & & 0.52 & & & 0.27 & 0.73 & OS7 \\
\hline
\end{tabular}

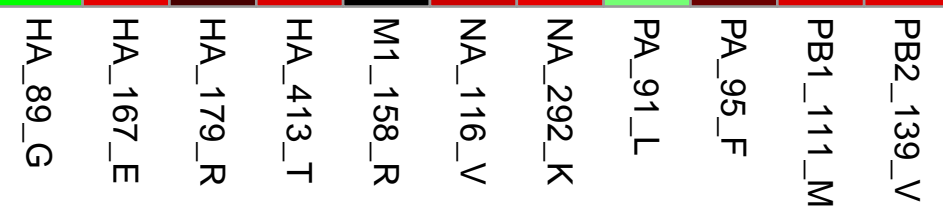

C

\begin{tabular}{|c|c|c|c|c|c|c|c|c|c|c|c|}
\hline 0.02 & 0.49 & 0.00 & 0.48 & 0.87 & 0.02 & 0.65 & 0.84 & 0.12 & 0.00 & 0.00 & POS \\
\hline 0.13 & & 0.80 & 0.91 & & 0.34 & 0.85 & NA & NA & NA & 0.81 & Zan1 \\
\hline 0.02 & & & & & NA & $0.8 s$ & NA & NA & NA & 0.80 & Zan2 \\
\hline 0.09 & & & & & 0.90 & & NA & NA & NA & 0.88 & Zan3 \\
\hline 0.05 & & & & & 0.91 & & & & 0.01 & & Zan4 \\
\hline 0.01 & & & & & 0.71 & 0.91 & & & NA & & Zan5 \\
\hline 0.08 & & & & & NA & 0.88 & & & NA & & Zan6 \\
\hline 0.13 & & 0.86 & 0.94 & 0.9 & 0.73 & 0.85 & 9 & 0.9 & NA & 0.87 & Zan7 \\
\hline 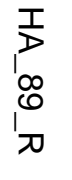 & $\begin{array}{l}\stackrel{I}{D} \\
\vec{D} \\
\stackrel{\vec{D}}{\mid} \\
\mathrm{m}\end{array}$ & $\begin{array}{l}\frac{T}{D} \\
\vec{V} \\
\vec{U} \\
-\end{array}$ & $\begin{array}{l}\frac{T}{D} \\
\frac{\Delta}{\omega} \\
I_{-1}\end{array}$ & 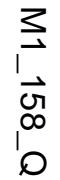 & $\begin{array}{l}Z \\
\text { D } \\
\overrightarrow{\vec{a}} \\
\vec{\sigma}\end{array}$ & $\begin{array}{l}Z \\
\text { סD } \\
\text { I } \\
\text { I } \\
\text { D }\end{array}$ & 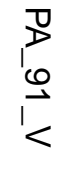 & 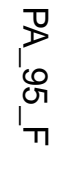 & $\begin{array}{l}\underset{0}{\varpi 0} \\
\underline{\mid} \\
\vec{\Xi} \\
\vec{\square}\end{array}$ & $\begin{array}{l}\text { W } \\
\text { N } \\
\vec{\omega} \\
\vec{\omega} \\
\end{array}$ & \\
\hline
\end{tabular}

B

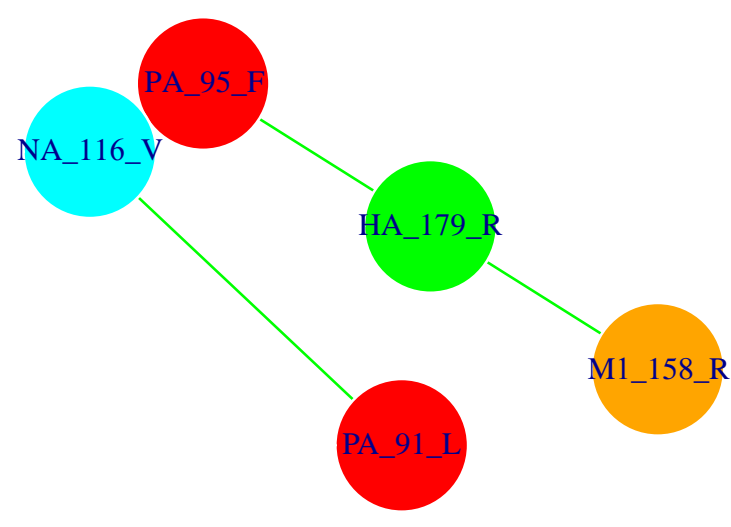

D

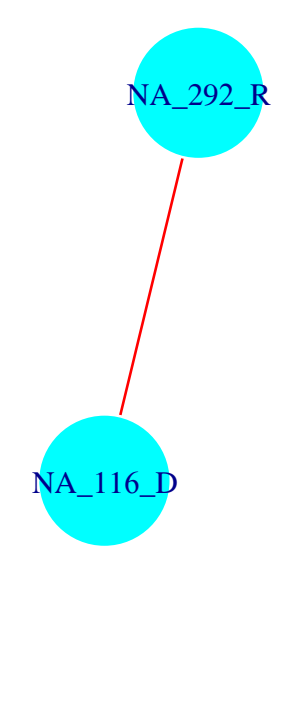


A N8 wild type

N8 Val1 16Asp

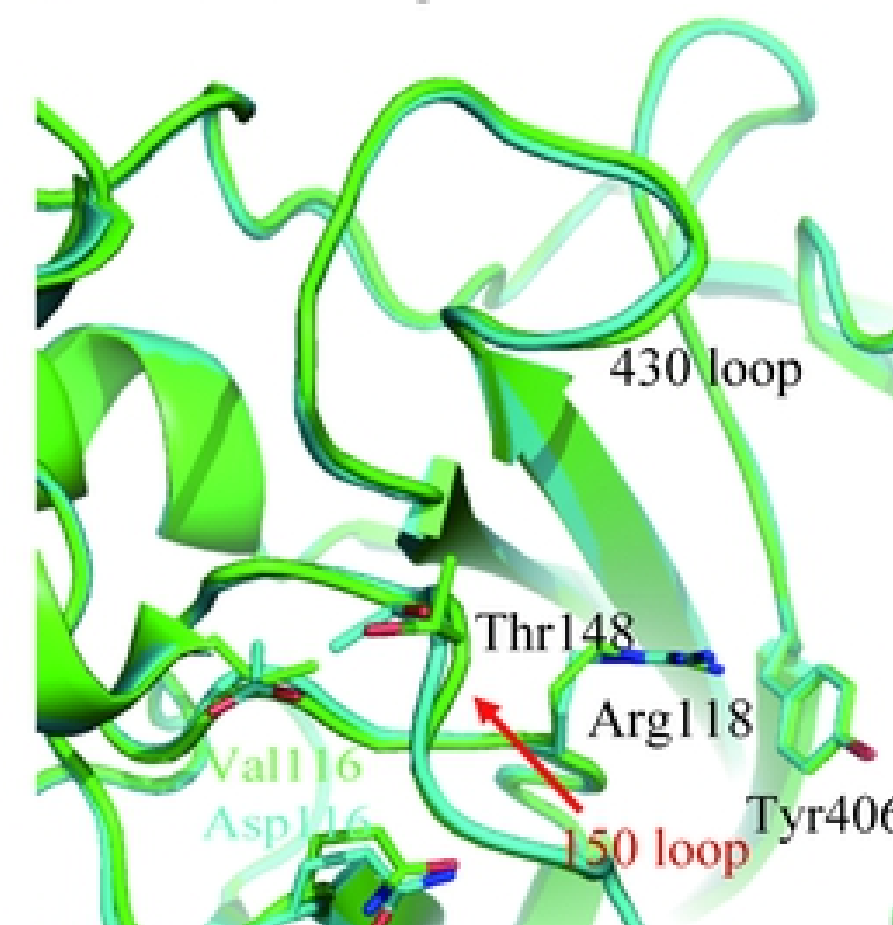

皮

P

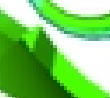

B

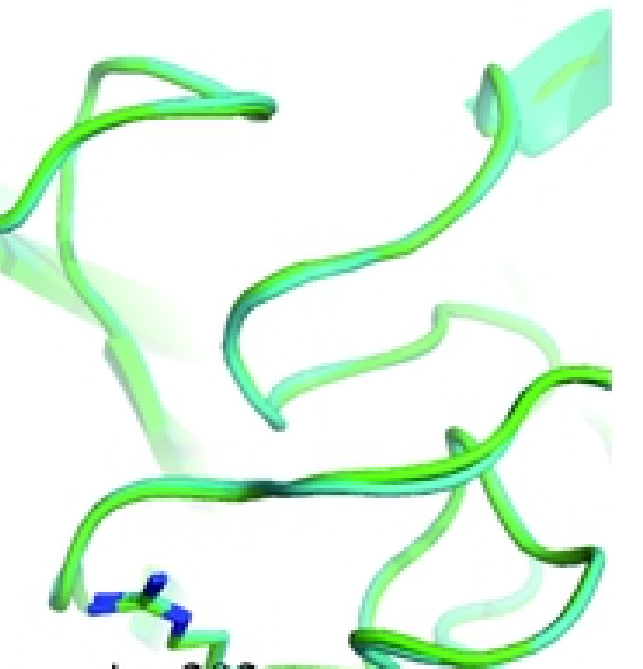

Arg 26
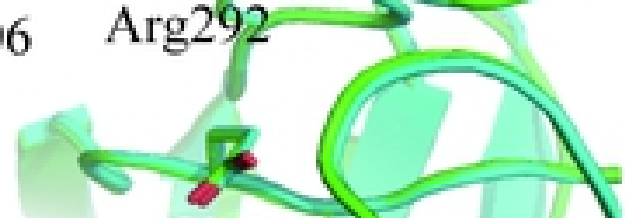
Glu276

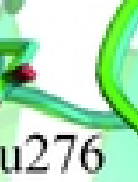

B $\simeq$ N8 wild type

N8 Arg292Lys
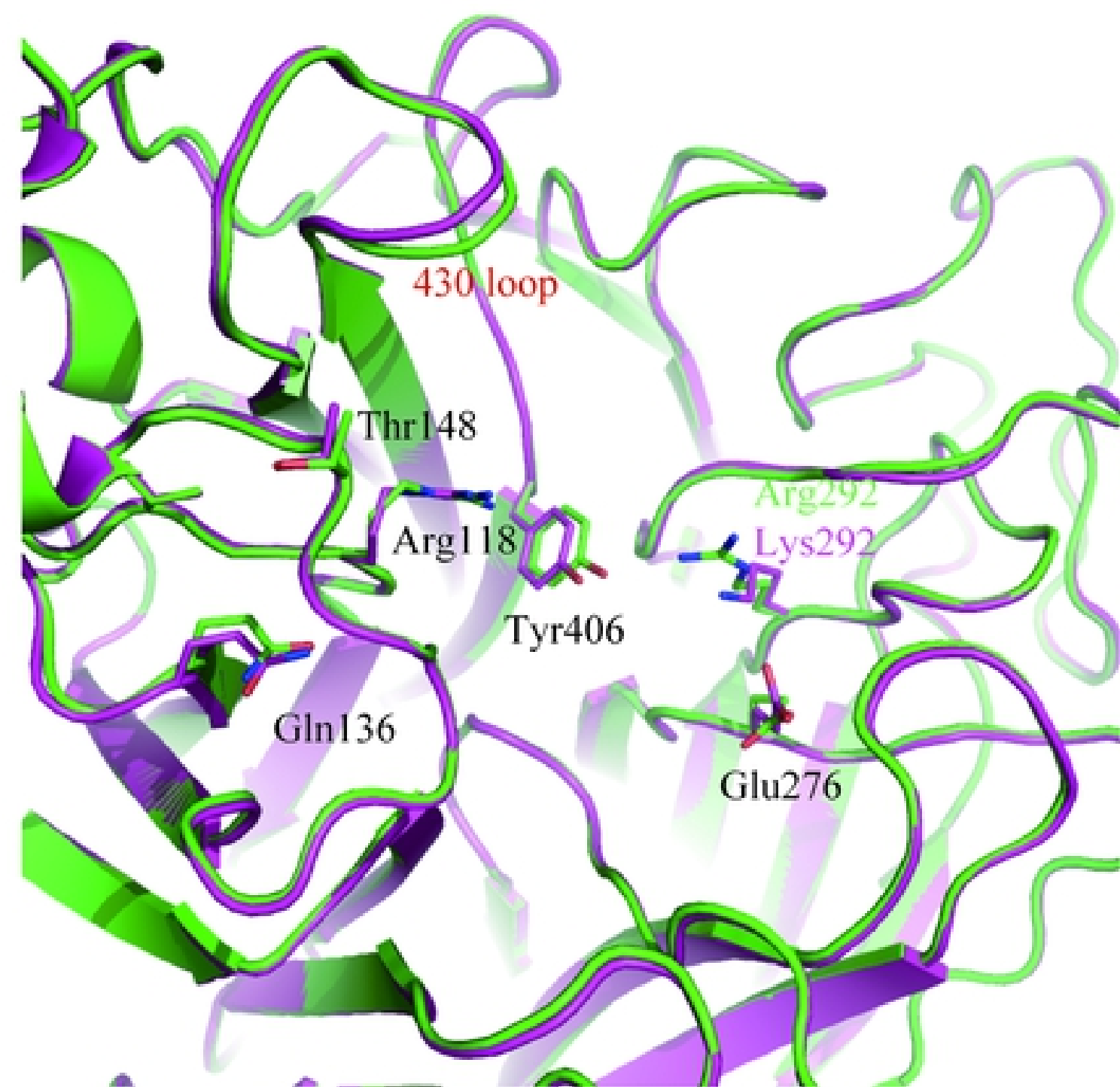

48
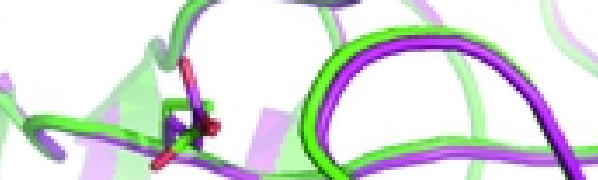

Glu276

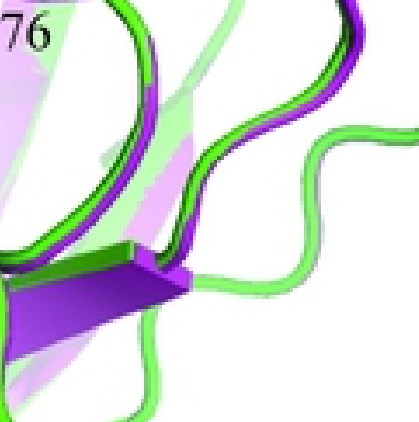


Ns wild sppe- Sostlamivir

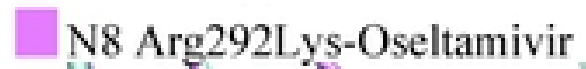

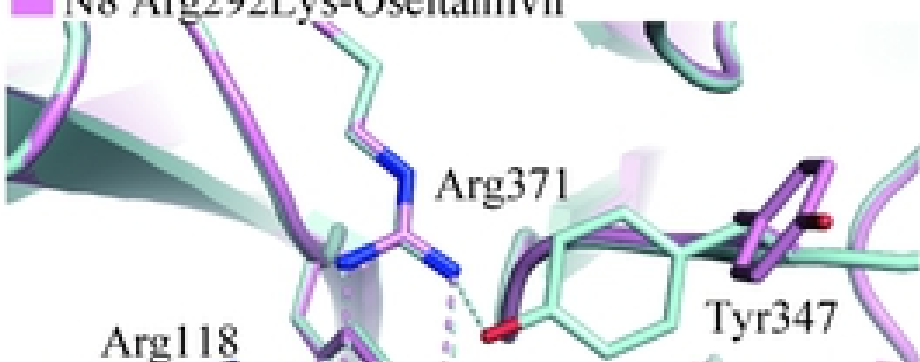

Arg1 18 $\sqrt{\mathrm{Y} 406} \cdot \mathrm{P}$

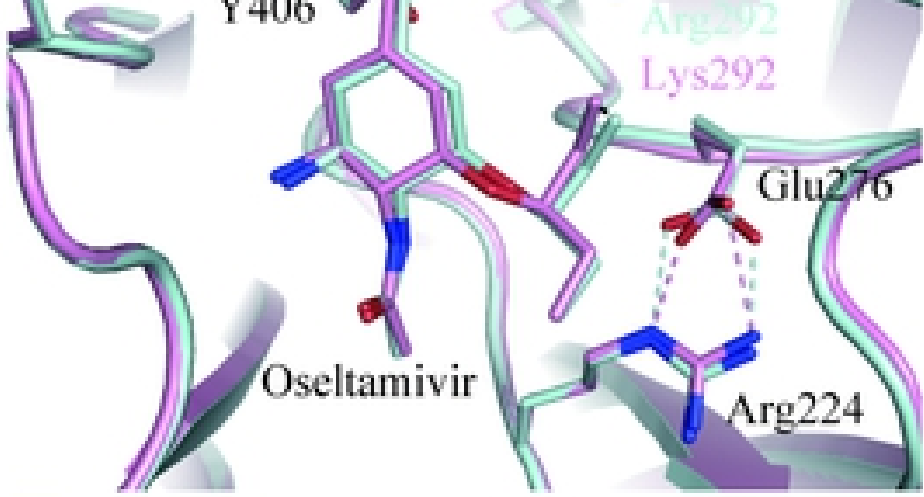

N8 wild type-Zanamivir

N8 Arg292Lys-Zanamivir

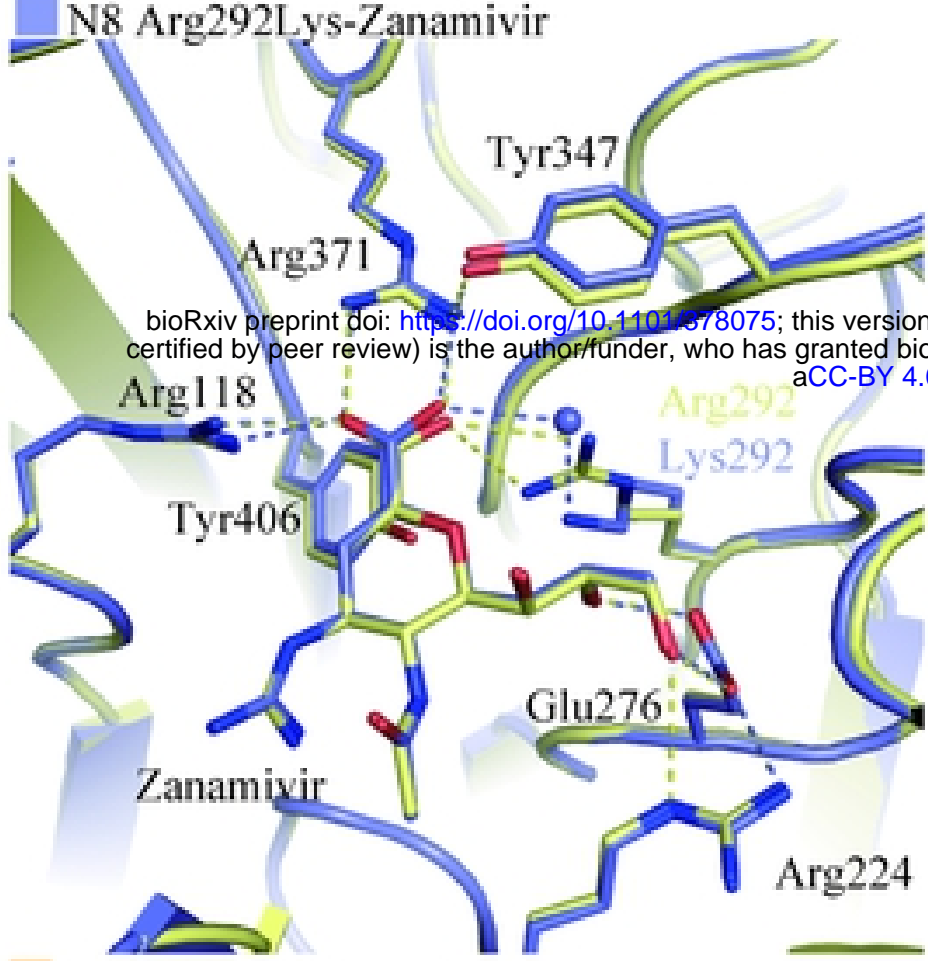

N8 wild type-Peramivir

N8 Arg292Lys-Peramivir

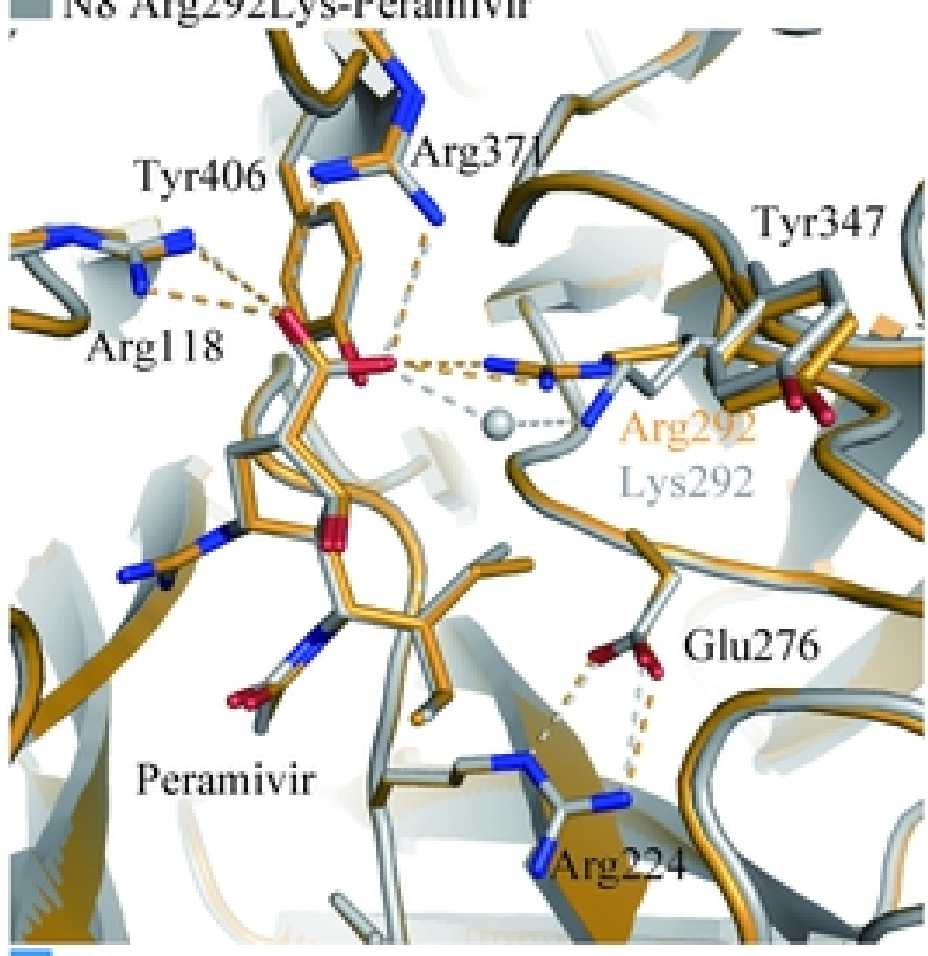

$\mathrm{G}$ N8 wild type-Laninamivir

N8 Arg292Lys-Laninamivir

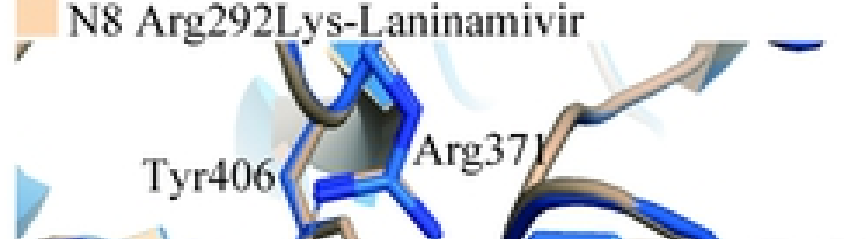

(a)

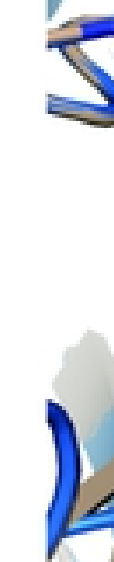

B

eltamivir

N8 Arg292Lys-Oseltamivir

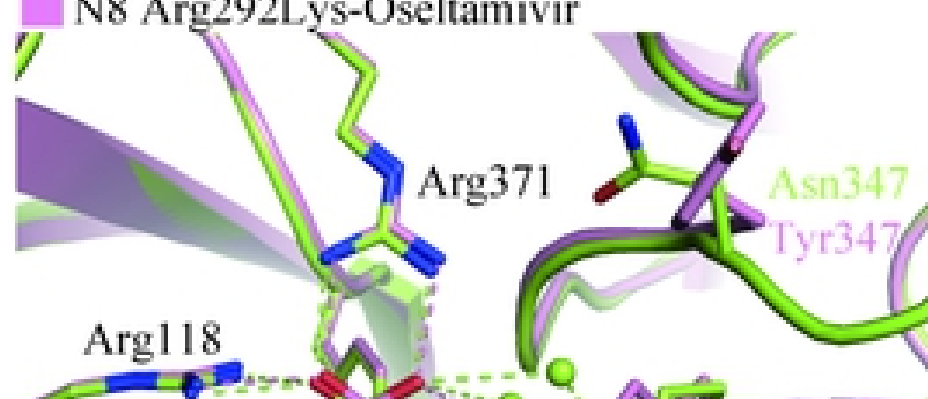

$\operatorname{Arg} 118$

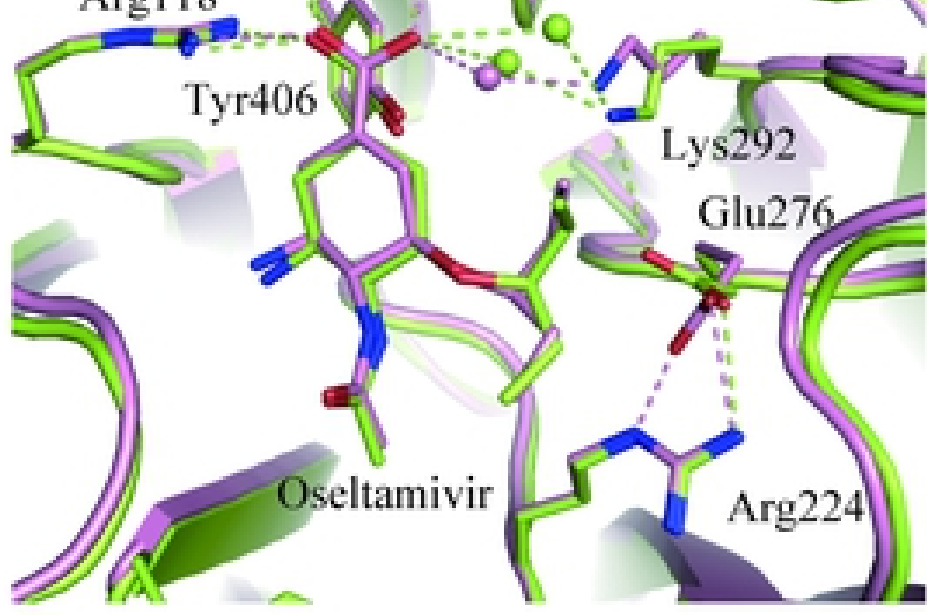

D N9 Arg292Lys-Zanamivir

N8 Arg292Lys-Zanamivir

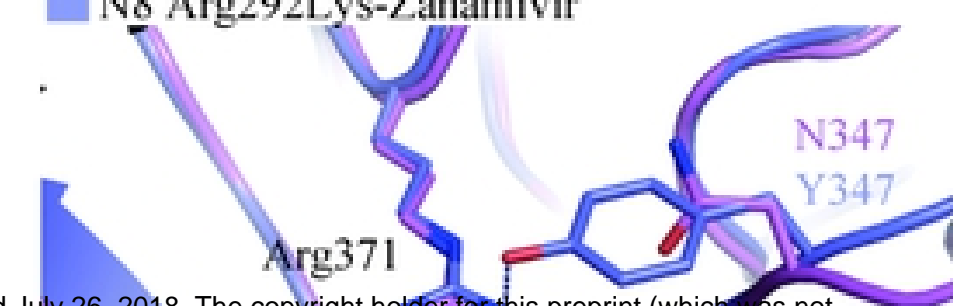

N347

r

as not

cense to display the preprint in perpetutity. It is made avaliable under
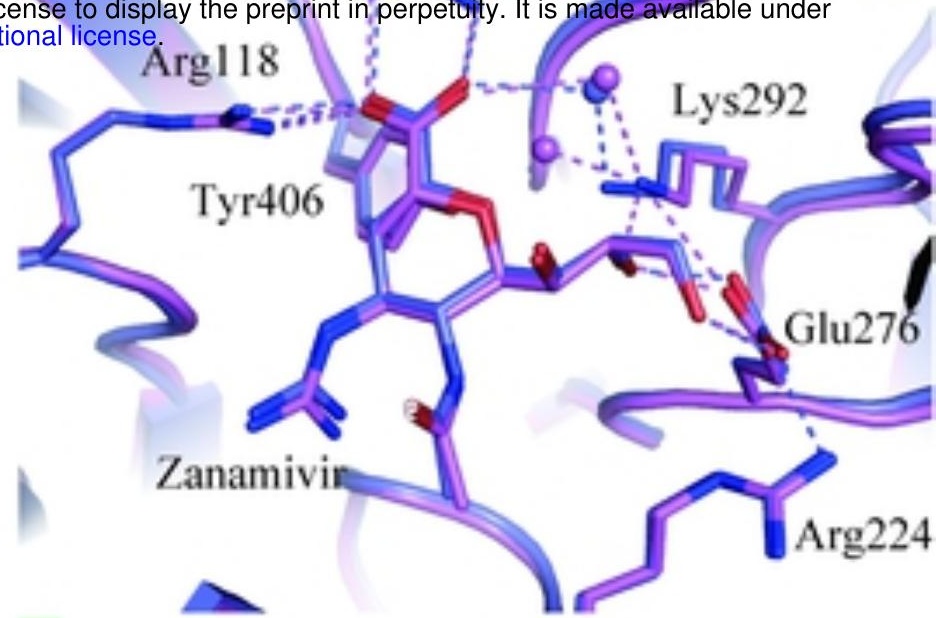

F N9 Arg292Lys-Peramivir

- N8 Arg292Lys-Peramivir

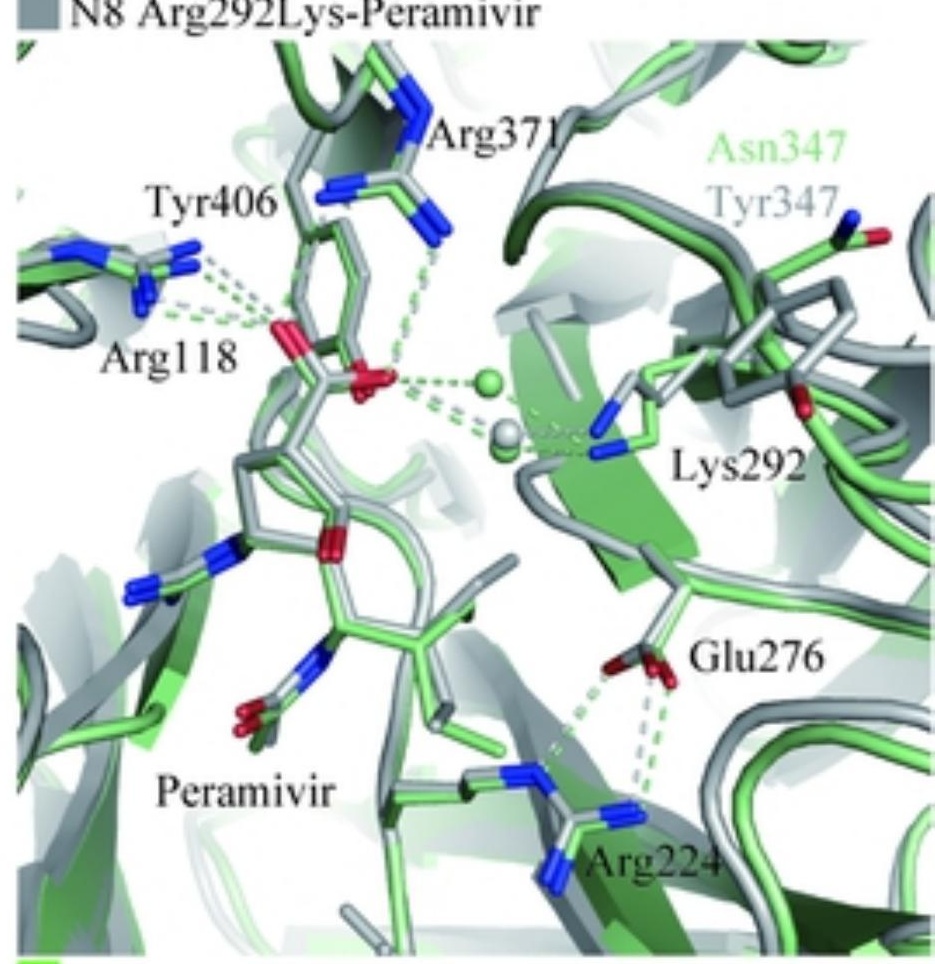

H N9 Arg292Lys-Laninamivir N8 Arg292Lys-Laninamivir

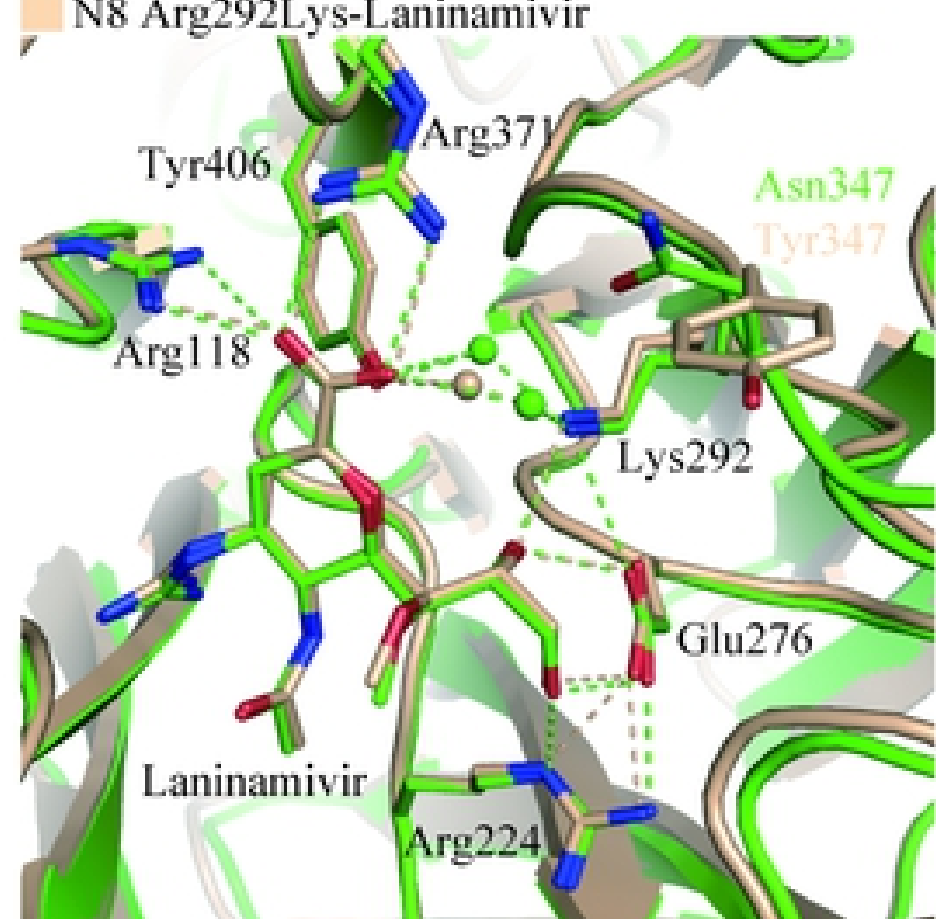


A N8 wild type-Oseltamivir

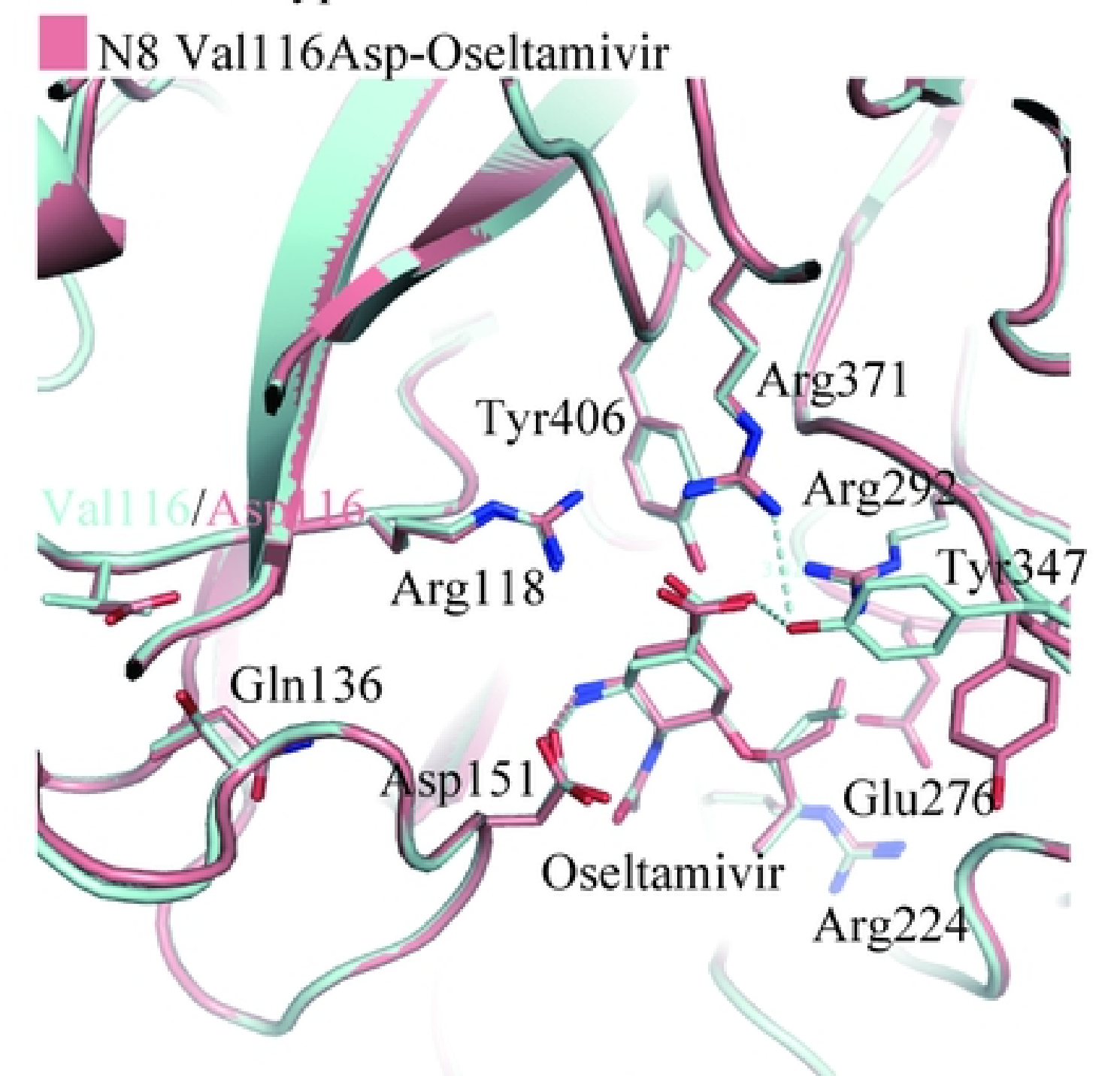

B N8 wild type-Zanamivir

N8 Val1 16Asp-Zanamivir
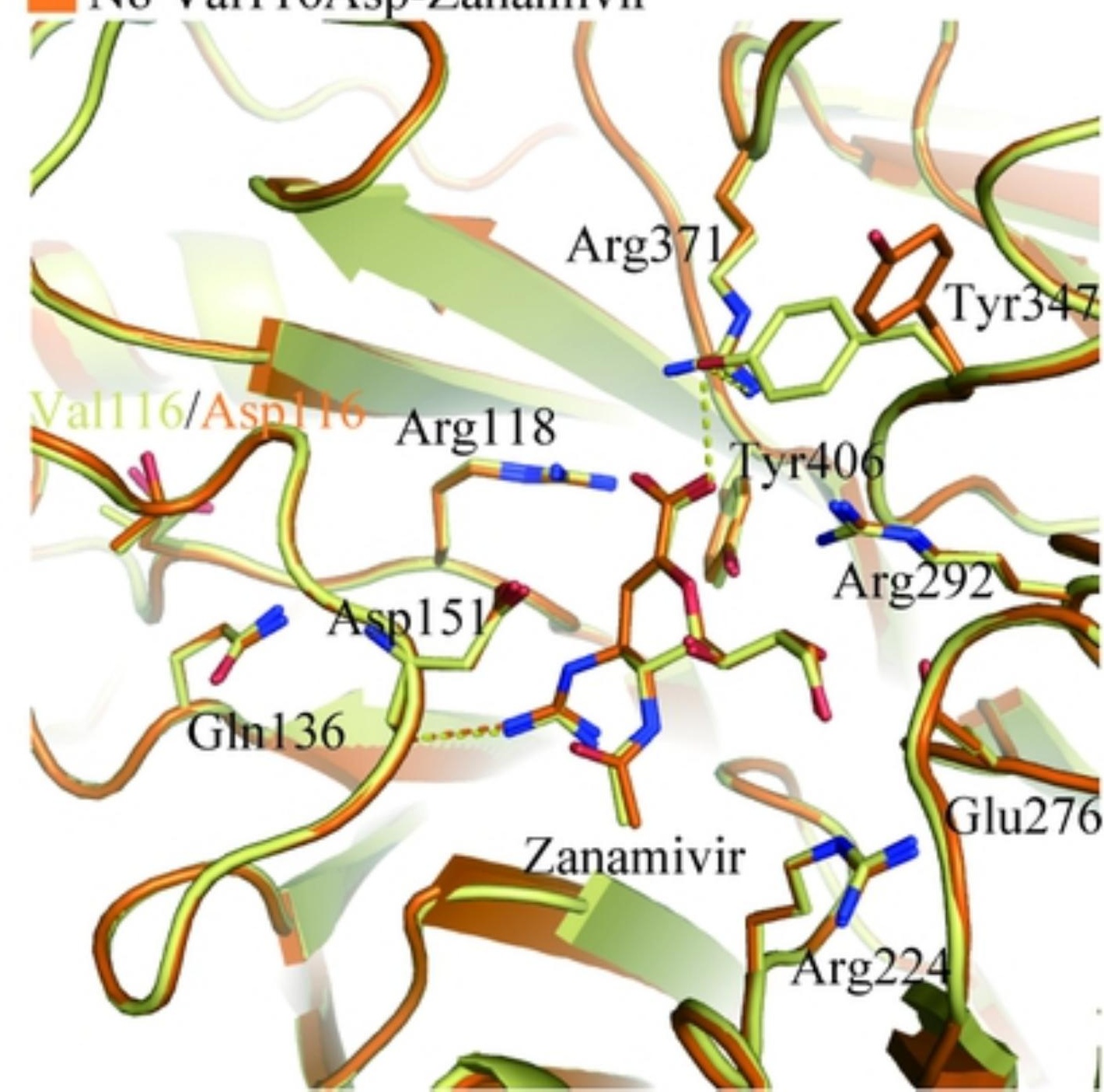